\title{
SINGLE-DOSE COLD MODIFIED BRETSCHNEIDER SOLUTION FOR MYOCARDIAL PROTECTION IN THE SURGICAL TREATMENT OF PATIENTS WITH AORTIC VALVE STENOSIS AND LEFT VENTRICULAR HYPERTROPHY
}

\author{
Petar H. Uzov', \\ Chavdar M. Ivanov ${ }^{1}$ \\ Department of Cardiac Surgery, \\ Heart and Brain Hospital - Pleven \\ ${ }^{1}$ Medical University - Pleven
}

\section{Corresponding Author:}

Chavdar M. Ivanov

Department of Cardiac Surgery, Heart and Brain Hospital - Pleven

2, Pier Curie Str.

Pleven, 5800

e-mail: shavdar@abv.bg

Received: June 06, 2021

Revision received: July 08, 2021

Accepted: November 25, 2021

\section{Summary}

Aortic valve stenosis (AS) is predominantly caused by degenerative calcification in patients over 70 years. It obstructs the left ventricular outflow tract. The result is concentric left ventricular hypertrophy (LVH) wall stress and ischemia-induced myocardial fibrosis. [1] According to the European Association of Cardiothoracic Surgery, all high-grade AS patients are indicated for Aortic valve intervention [2]. One of the most recommendable methods for myocardial protection during surgical aortic valve replacement is antegrade delivery of cold crystalloid cardioplegia. Our retrospective study included 173 operated patients with AS and LVH. We observed the MB fraction of the enzyme creatine phosphokinase (CPK-MB) as a postoperative marker for myocardial hypoprotection in different patients. Our results showed that a single dose of modified Bretschnaider solution provided safe and long enough myocardial protection during conventional and minimally invasive aortic valve replacement in patients with AS and LVH [3].

Keywords: antegrade cardioplegia, Bretschneider solution, myocardial protection

\section{Introduction}

Surgical aortic valve replacement under cardiac arrest is the approved treatment in patients with AS [2].

Application of HTK (Histidine, Tryptophan, Ketoglutarate) solution was reported by Bretschneider in the 1970s [4]. It is classified as intracellular, crystalloid cardioplegia due to its low sodium and calcium content. Sodium depletion of the extracellular space causes a hyperpolarization of the myocyte plasma membrane, inducing cardiac arrest in diastole. The mechanism of action differs from conventional 'extracellular' cardioplegic solutions with high potassium content and leads to cardiac arrest by membrane depolarization [5] (Table 1).

The high histidine content buffers the acidosis caused by the accumulation of anaerobic metabolites during the long ischaemic period, ketoglutarate improves adenosine triphosphate production during reperfusion, tryptophan stabilizes the cell membrane, and mannitol decreases cellular edema and acts as a 
Table 1. Composition of a modified Bretshneider solution

\begin{tabular}{cc}
\multicolumn{2}{c}{ Modified Bretshneider solution } \\
\hline $\mathrm{Na}^{+}$ & $15 \mathrm{mmol} / \mathrm{l}$ \\
\hline $\mathrm{K}^{+}$ & $9 \mathrm{mmol} / \mathrm{l}$ \\
\hline $\mathrm{Mg}^{2+}$ & $4 \mathrm{mmol} / 1$ \\
\hline $\mathrm{Ca}^{2+}$ & $0.015 \mathrm{mmol} / \mathrm{l}$ \\
\hline Histidine & $198 \mathrm{mmol} / 1$ \\
\hline Triptophan & $2 \mathrm{mmol} / 1$ \\
\hline Ketoglutarate & $1 \mathrm{mmol} / 1$ \\
\hline Mannitol & $30 \mathrm{mmol} / \mathrm{l}$ \\
\hline $\mathrm{pH}$ & $7.02-1.20$
\end{tabular}

free-radical scavenger [6].

The data published confirming its efficacy among patients with LVH is scarce.

\section{Materials and Methods}

We performed a single-center, retrospective study on 173 consecutive elective patients with surgical aortic valve replacement (AVR) for isolated AS with preserved ejection fraction (EF) and LVH. All patients were operated on in our institution between April 2017 and October 2020. Preoperative transthoracic echocardiography and intraoperative transesophageal echocardiography were performed to complete criteria for AS and LVH. The study has no control group with other types of cardioprotection or without.

According to our institutional protocol, all the patients underwent replacement of aortic valve under cardiopulmonary bypass and cardiac arrest, achieved by antegrade admission of single-shot cold $\left(5-7^{\circ} \mathrm{C}\right)$ crystalloid cardioplegia - modified Bretschneider solution at a dose of $20 \mathrm{ml} / \mathrm{kg}$ for a minimum of 6-minute admission time.

The patients were divided into two groups: one group with minimally invasive aortic valve replacement (mini AVR) and another group with conventional aortic valve replacement (AVR). The following parameters - sex, age, crossclamp time, operative time, body mass index, ejection fraction for both groups- were observed to find any correlation of each of them with postoperative CPK-MB.

The postoperative parameters we observed were intensive care unit (ICU)- stay and duration of mechanical ventilation as indicators of the patients' overall state and recovery speed.

\section{Aim}

We used the results obtained to estimate the safety and efficiency of the method applied in our institution for cardioplegia in patients with $\mathrm{AS}$ and LVH.

Our second aim was to observe possible differences in myocardial protection in patients with minimally invasive aortic valve replacement and conventional aortic valve replacement.

\section{Result}

\section{Patients}

We studied 173 patients (89 males and 84 females). The median age of the patients was 69.5 years, standard deviation $(\mathrm{SD}=8.9$ years, range 32 - 89). Depending on the operative

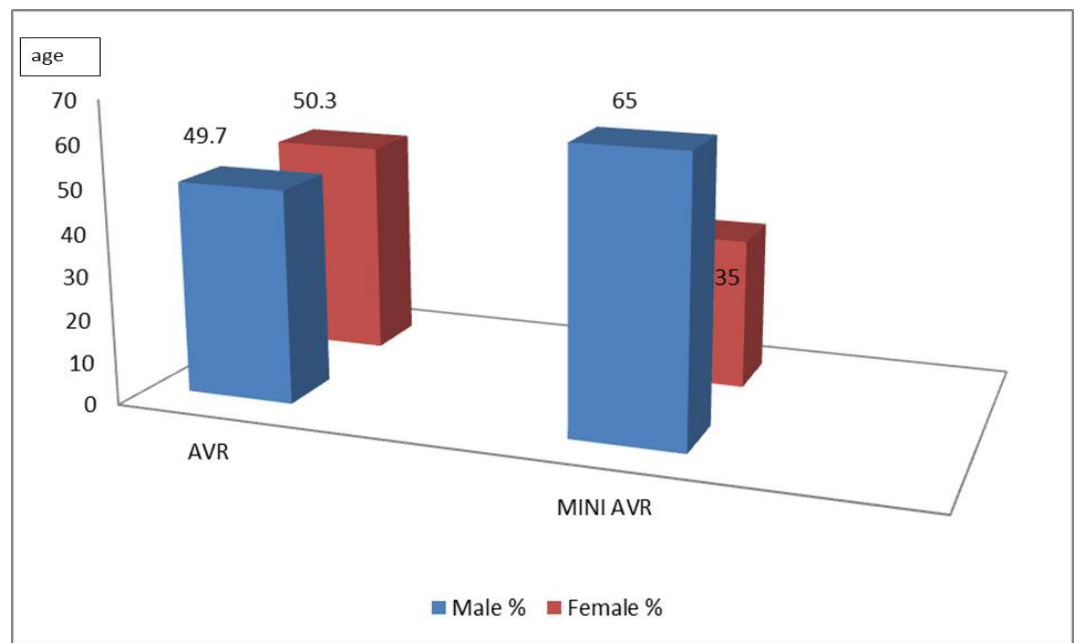

Figure 1. Demographic distribution 
incision, they were divided into two groups.

The group treated with conventional total sternotomy included 153 patients ( 76 males and 77 females, median age 69.7 years, $\mathrm{SD}=8.9$, and age range 32 - 89).

The second group was treated by minimally invasive aortic valve replacement (mini AVR). This operation was performed through a less than 7-centimeter skin incision and partial upper J-shaped sternotomy. This group included

The mini AVR group included 20 patients: 13 males and seven females, median age 67.8 years, $\mathrm{SD}=8.4$ years, age range $48-82$ ).(Figure 1)

Patients in both groups had preserved LV systolic function. The average EF in the AVR group was $57 \%(\mathrm{SD}=11 \%)$. The average $\mathrm{EF}$ in the Mini AVR group was $56 \%$ (SD 12\%). ( Figure 2)

The operative time was shorter in the mini AVR group. The average duration of the surgical procedure in the AVR group was 204 minutes $(\mathrm{SD}=56 \mathrm{~min})$. The average duration of the surgical procedure in the mini AVR group was 173 minutes $(\mathrm{SD}=36 \mathrm{~min})$. Cross-clamp ischemic times were similar for all patients in both groups. The average cross-clamp time in the AVR group was 64 minutes $(\mathrm{SD}=20 \mathrm{~min})$, while in the mini AVR group, it was 57 minutes ( $\mathrm{SD}=10 \mathrm{~min}$ ).(Figure 3 )

We measured the body mass index (BMI) as well. The average BMI in the AVR group was $28 \mathrm{~kg} / \mathrm{m}^{2}\left(\mathrm{SD}=5 \mathrm{~kg} / \mathrm{m}^{2}\right)$. The average BMI in the mini AVR group was $27 \mathrm{~kg} / \mathrm{m}^{2}\left(\mathrm{SD}=4 \mathrm{~kg} / \mathrm{m}^{2}\right)$.

The MB fraction of creatine phosphokinase is an intracellular enzyme special for myocardial cells. Its serum level correlates with perioperative cellular death. Postoperative CPK-MB on the second postoperative day is a well-known indicator for perioperative myocardial ischemia [15]. The average postoperative level of CPK$\mathrm{MB}$ in the AVR group was $65 \mathrm{U} / \mathrm{L}(\mathrm{SD}=72$ $\mathrm{U} / \mathrm{L})$. For the mini AVR group, it was $47 \mathrm{U} / \mathrm{L}$ $(\mathrm{SD}=18 \mathrm{U} / \mathrm{L})$. (Figure 4$)$

\section{$\mathrm{EF} \%$}

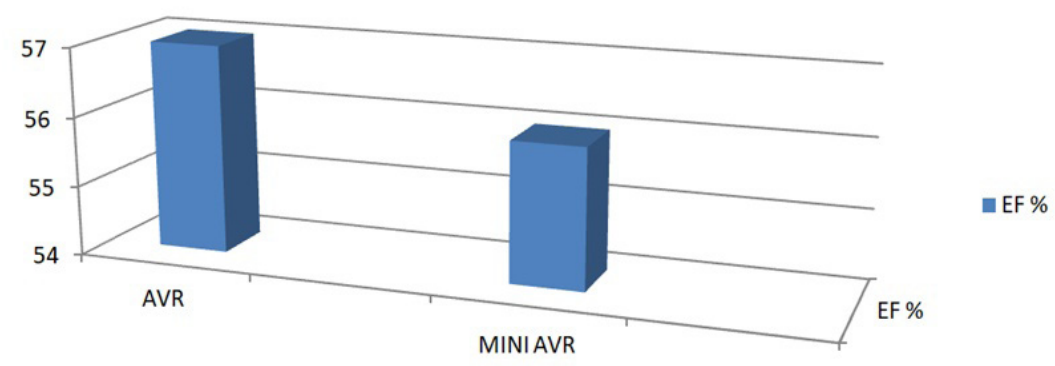

Figure 2. Mean EF in both groups

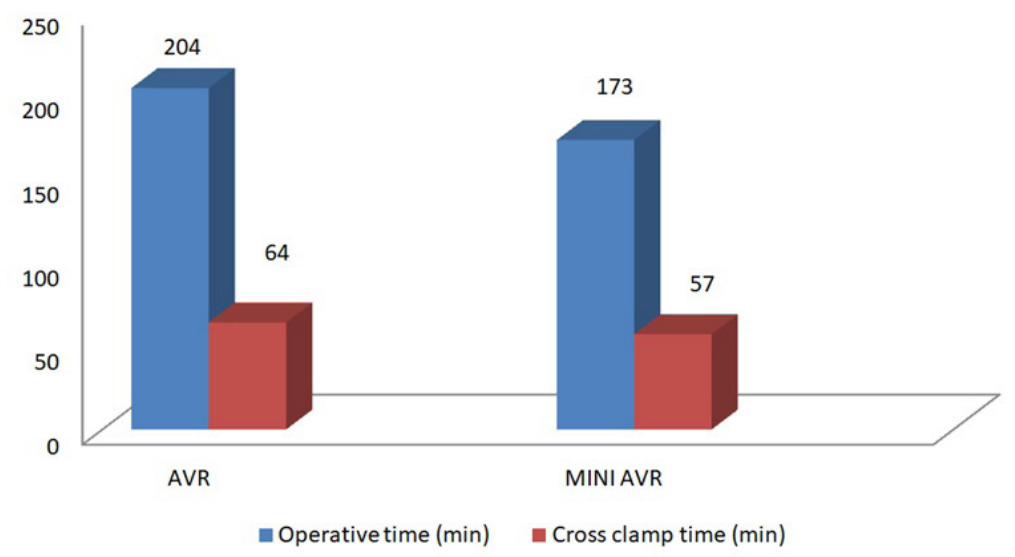

Figure 3. Comparison between operative time and cross clamp time in the two groups 
ICU stay is a criterion that represents the speed of a patient's recovery. In the AVR group, it was 79 hours $(\mathrm{SD}=78 \mathrm{~h})$. The average duration for the mini AVR group was 70 hours ( $S D=35 \mathrm{~h}$ ). Another parameter in our study was the postoperative time of invasive lung ventilation (ILV). The average ILV time in the AVR group was 19 hours $(\mathrm{SD}=40 \mathrm{~h})$. The average ILV time in the mini AVR group was 15.6 hours $(\mathrm{SD}=12$ h.).(Figure 5)

The average in-hospital mortality in the AVR and Mini AVR groups was 5.2\% and $0 \%$, respectively.

\section{Statistical data analysis}

Based on the descriptive statistical analysis for both groups, we conclude that frequency distribution of age, EF, cross-clamp time, and operative time were defined as "normal" because the values were very similar to the Central Tendency indicators. Such a statement was not confirmed for the CPK-MB, time in ICU, and duration of mechanical ventilation. This is defined as a right-skewed positive distribution. (Table 2)

The statistical processing of the result was made using the statistical package SPSS of IBM Corporation. Results with a significant coefficient $\mathrm{p}<0.05$ were considered statistically reliable. The following methods were used for data analysis: Spearman's rank correlation coefficient; Regression correlation analysis; classification tree; Dispersion Analysis ANOVA; Independent samples t-test and Chi-square.

We evaluated the strength and direction of all possible correlations through coefficient rank correlations of the Spearman test for all indicators in both groups.

A high level of strength and positive correlation was found between operative time and cross-clamp time indicators $(r=0.755$, $\mathrm{p}=0.000$ ).

A moderate level of strength and positive correlation was found between the three pair

\section{MB fraction U/L}

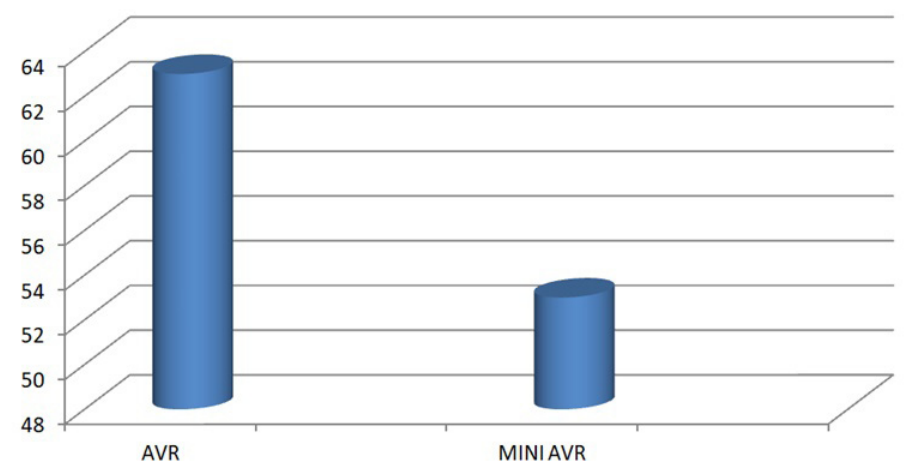

Figure 4. MB fraction in both groups

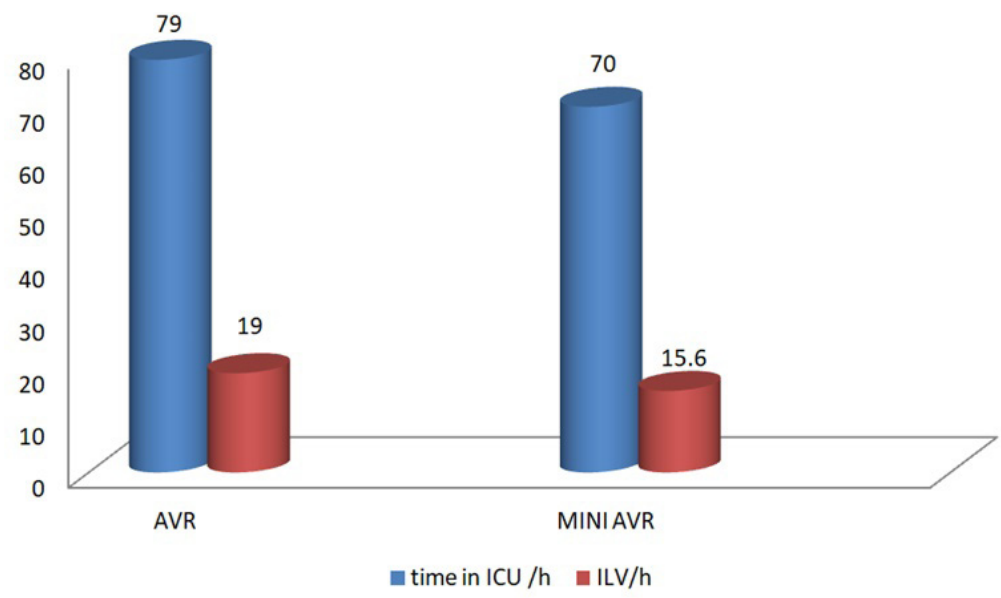

Figure 5. Comparison between ICU-time and ILV-time in the two groups 


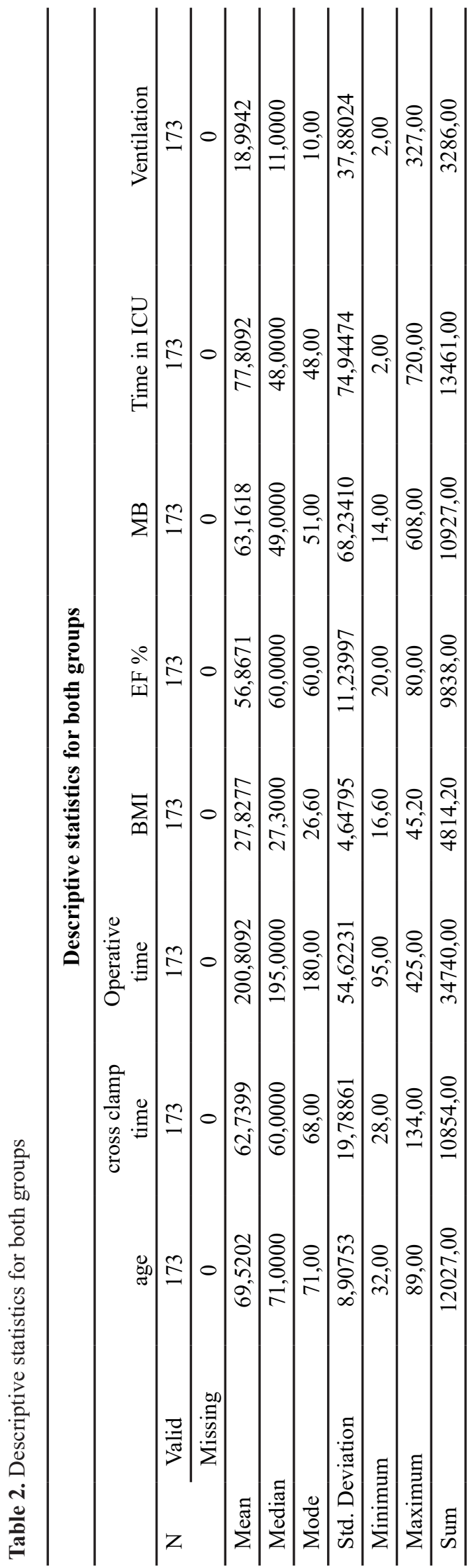

indicators: operative time and $\mathrm{MB}$ fraction $(\mathrm{r}$ $=0.333 ; \mathrm{p}=0.000)$; cross clamp time and $\mathrm{MB}$ fraction $(\mathrm{r}=0.342 ; \mathrm{p}=0.000)$ and between time in ICU and ILV time $(r=0.360 ; p=0.000)$

A low level of strength and negative correlation was established between $\mathrm{EF}$ and mortality rate $(\mathrm{r}=-0.150 ; \mathrm{p}=0.049)$ [13].

Low level of strength and positive correlation is available between the three pair indicators: time in ICU and Mortality rate $(\mathrm{r}=0.71$; $\mathrm{p}=0.025)$, ILV time and Mortality rate $(\mathrm{r}=0.204$; $\mathrm{p}=0.007)$ and BMI and time in ICU $(\mathrm{r}=0.150$; $\mathrm{p}=0.048)$. ( Table 3 )

Similar trends with similar strength were seen in the AVR group, while in the mini AVR group, we found only one correlation between cross-clamp time and operative time. The link was strong, and the direction was positive.

Using regression-correlation analysis, we found a significant link between the EF and the time in ICU. We find a low level of strength and negative correlation: $3.1 \%$ of the differences in time in ICU were due to EF, which means that if EF increased by $1 \%$, the time in ICU decreased by 1.18 hours $(\mathrm{p}=0.021)$.

Base on the same correlation analysis, we discovered a significant link between the factor cross-clamp time and the result postoperative MB fraction. The correlation link was less strong, of positive direction, and $5.7 \%$ from the differences in MB fraction were attributable to cross-clamp time. If the cross-clamp time increased by 1 min, then the MB fraction increased by $0.82 \mathrm{U} / 1$. $(\mathrm{p}=0.002)$.

We discovered another significant link between the operative time and MB fraction. The correlation link was weak, of positive direction, and $5.4 \%$ of the differences in MB fraction were due to operative time. If operative time increased by 1 minute, MB fraction increased by 9 U/1 (p $=0.02$ )

Another significant link was found between the cross-clamp time and the operation time. The correlation link was very strong, of positive direction, and $54 \%$ of the differences in operation time were due to cross-clamp time. If crossclamp time increased by $1 \mathrm{~min}$, the operative time was increase by 2.03 minutes longer.

Because of a significant link, the ILV time increases by 1.31 minutes if BMI increases by 1 $\mathrm{kg} / \mathrm{m}^{2}$, which means that $2.6 \%$ of the differences 


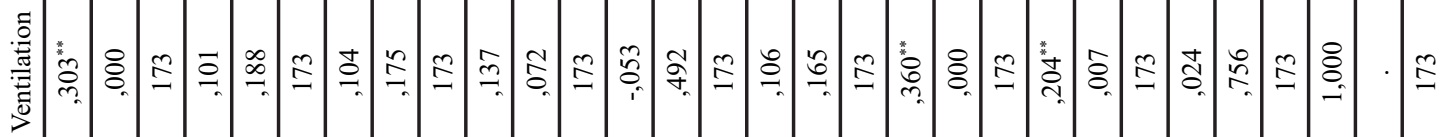

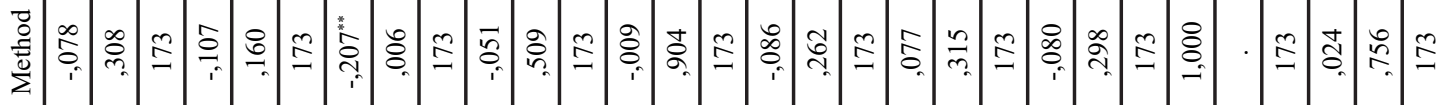

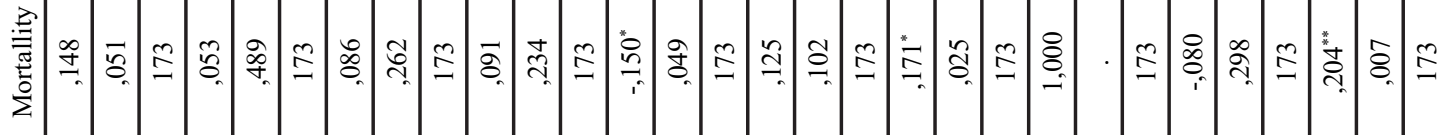

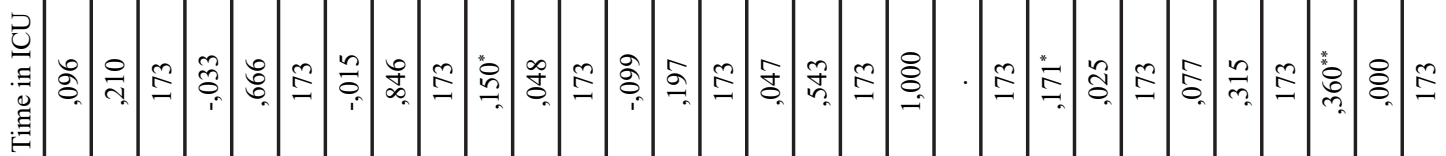

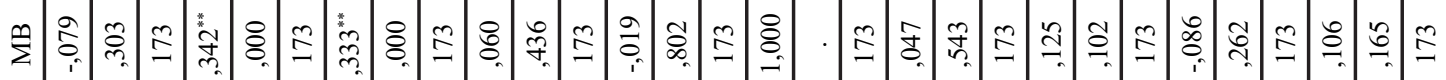

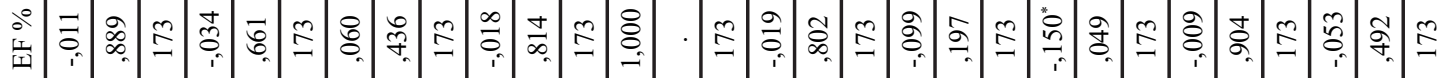

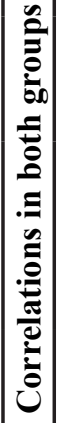

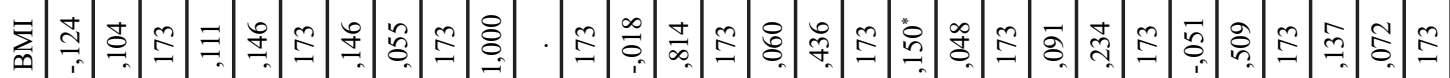

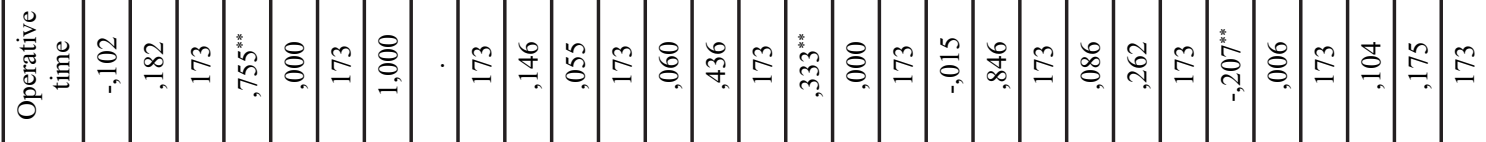

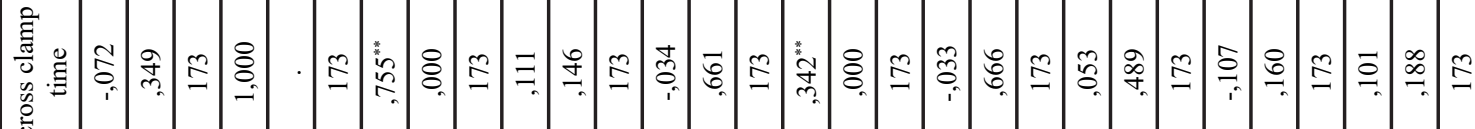

$\underbrace{}_{0}$

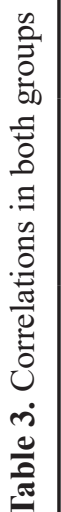

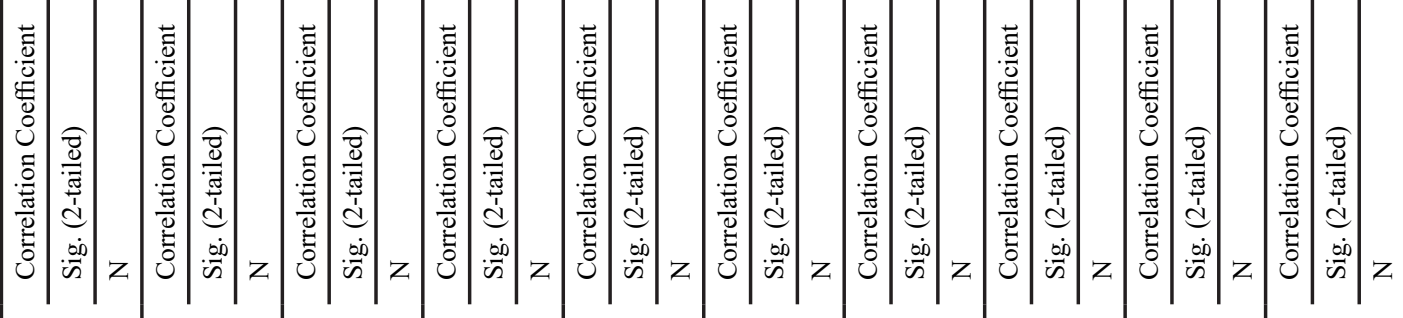

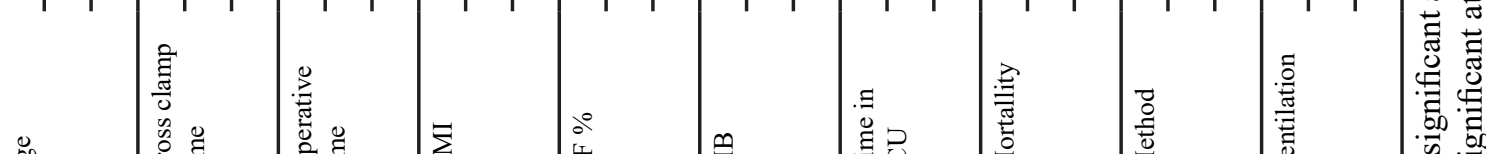

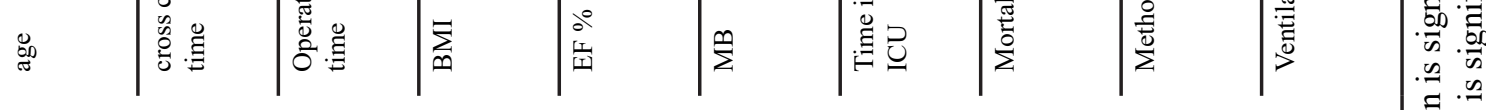

离

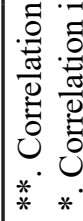


in ILV time were due to BMI values $(\mathrm{p}=0.000)$ in our study.

Using the classification tree analyzing method for all patients, we established a significant link between ILV time as a factor and time in ICU as a result. This method allows for dividing the patients into two other groups: patients with ILV time equal to or less than 14 hours and patients with ILV time more than 14 hours. In the first group, we noticed that if the ILV time was less than 14 hours, the average ICU stay was almost two times lower than in the other group $(\mathrm{p}=0.009)$ (Figure 6). Such an observation supports the mini AVR operative method with a lower average ILV time. (Figure 6)

We used the classification tree method to investigate $\mathrm{EF}$ as a factor and mortality rate as a result. We found dependence between them. All of the lethal cases had an EF lower than $59 \%$. There was no mortality in the rest of the cases with $\mathrm{EF}$ above $59 \%(\mathrm{p}=0.036)$. (Figure 7)

We established a significant dependence between $\mathrm{EF}$ and mortality rate $(\mathrm{p}=0.044)$ with the independent samples t-test.

Dispersion Analysis ANOVA found a very close to a significant link between the operative method and operative time with a P-value of 0.08 .

If we use P-value -0.08 as a significant and consider the risk for error as $8 \%$, we can conclude that $2 \%$ of the differences in the operative time are due to the surgical method.

Using the chi-square test, we investigated the link between the operative method as a factor and

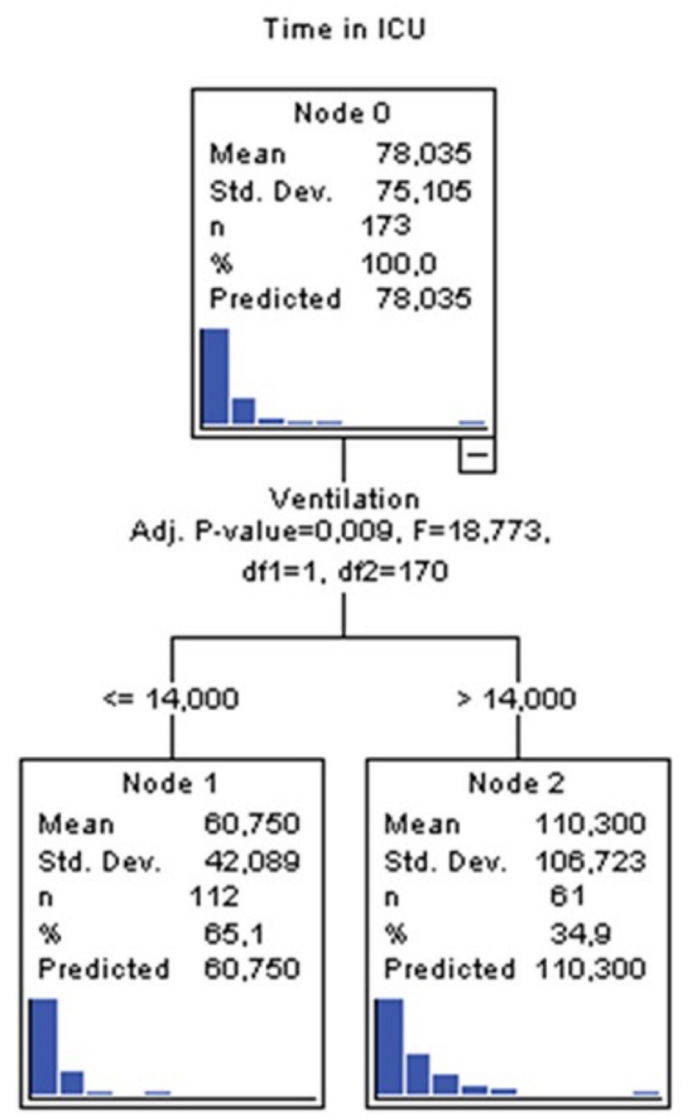

Figure 6. Classification tree- ILV - time (factor) and time in ICU (result)

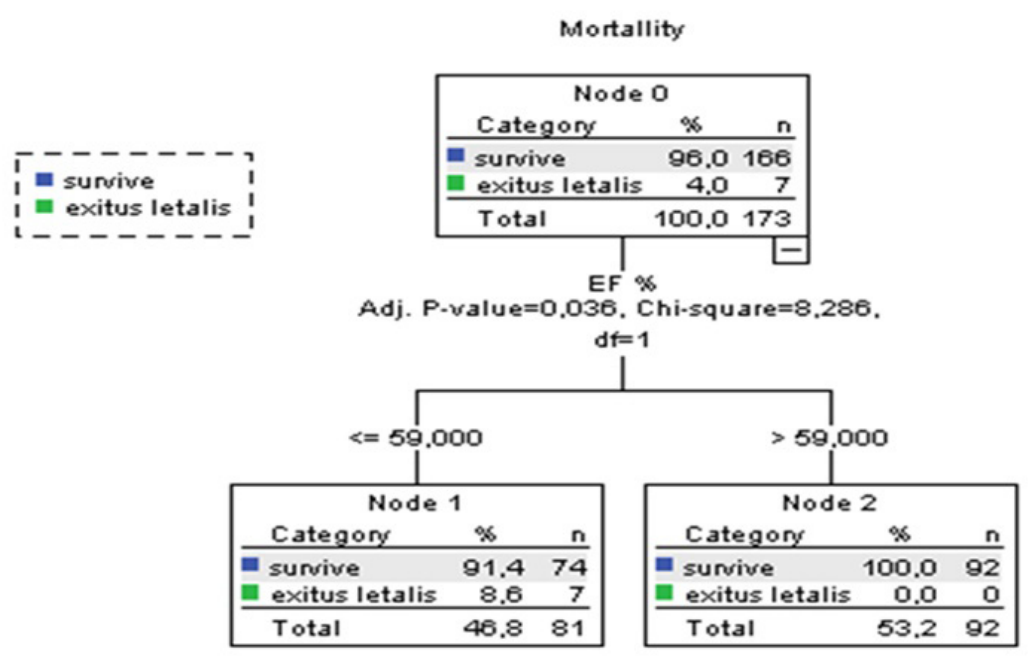

Figure 7. Classification tree - EF (factor) and Mortality rate (result) 
the mortality rate. We did not find a significant relationship between the two.

Although we did not prove a statistical significance between the operative method and mortality rate in the investigated groups, we observed no fatal cases in the mini AVR group and a $5.2 \%$ mortality rate in the AVR group, i.e., mini AVR was as safe as conventional AVR.

\section{Discussion}

Fannelop reported a study supporting the application of the Bretschneider solution. The study was carried out on pigs. HTK and intermittent cold-blood cardioplegia was administrated in different groups of animals. The conclusion was that HTK provides better myocardial protection and fewer postoperative cardiac incidents.

G. Scrascia et al. studied 112 patients divided into two groups. They reported less myocardial enzyme relief in patients with longer crossclamp time ( $>150 \mathrm{~min})$, protected with Modified Bretschneider solution versus cold-blood cardioplegia. Our study observed decreasing the markers for myocardial necrosis in the patients with shorter operative times and cross-clamp times [8].

Careaga et al. found a significant correlation between patients protected with Modified Bretschneider and extracellular, crystalloid cold cardioplegia. In this study on thirty patients and an average cross-clamp time of $60 \mathrm{~min}$ in the Bretschneider group, they observed lower inotropic support and lower incidence of arrhythmia [9].

An interesting report was made by Liu et al., observing an apoptosis index measured after myocardial biopsy in patients protected with HTK and conventional extracellular cold cardioplegia. The result was the absence of apoptosis in the HTK group [10].

Right ventricular function was also observed by Haddad et al. They compared administration of HTK and warm blood cardioplegia in patients with mitral valve lesions and lower rightventricular ejection fractions. The result for the HTK group was lower cardiac volumes, higher pulmonary pressure, and longer ICU time [11].

James et al. carried out a large systematic study on 2114 patients and compared different types of cardioplegia with HTK. They did not find differences in mortality rates between the HTK and other groups. Also, these authors did not report a significant difference in $\mathrm{MB}$ fraction and postoperative low cardiac output syndromes in the groups. These results confirmed the safety of HTK compared with conventional cardioplegia.

We found only one study - that of Scrascia et al., comparing the efficacy of HTK in minimally invasive AVR and conventional AVR. However, in their conventional AVR group, blood cardioplegia was administrated.

A closer look at the myocardial enzyme relief due to the efficacy of the HTK solution reveals a
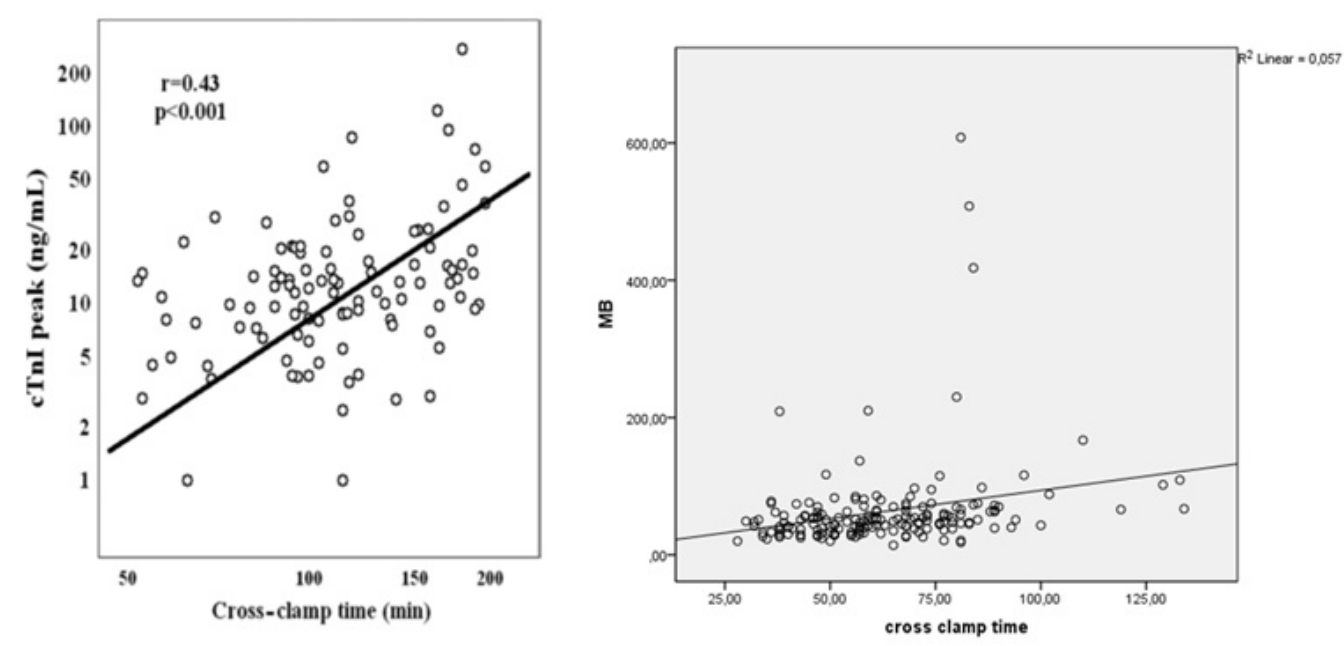

Figure 8. Graph of regression - Linear correlation of posoperative cardiac enzymes - Troponin (Scrascia study), MB fraction peacs (our study) and cross clamp time 
statistically positive linear correlation according to Scrascia and our study. (Figure 8) Some authors have not reported differences in MB relief between cases with HTK and conventional cardioplegia $[14,15]$.

Our observations support the finding of Seanson, i.e., that modified Bretschneider solution is effective cardioplegia, especially in minimally invasive surgery. We established that HTK cardioplegia in combination with shorter cross-clamp time (mini AVR), shorter operative time (mini AVR), and EF above 59\% results in shorter intensive care time (mini AVR), shorter invasive lung ventilation time (mini AVR), myocardial enzyme relief (mini AVR), and lower mortality rate (mini AVR).

Although we did not prove a significant statistical link between the two operative methods, we have enough data to describe the efficacy of modified Bretschneider solution in patients with aortic valve stenosis and LVH. Antegrade administration of cold, single-dose cardioplegia $(20 \mathrm{ml} / \mathrm{kg})$ is enough to protect the myocardium during the entire cross-clamp time.

Single-dose cold cardioplegia simplifies the surgical procedure, while a multi-dose is timeconsuming and may cause coronary ostia injury.

\section{Conclusion}

Our institutional protocol for myocardial protection with modified Bretschneider solution is an excellent choice for cardioprotection during surgical treatment of aortic valve stenosis with myocardial hypertrophy.

Better results can be achieved in the group with mini AVR, but they are not statistically significant due to the low number of patients we investigated.

The published data review and our study confirm that HTK solution is reliable cardioplegia for conventional use in all patients with AS and LVH.

\section{References}

1. Brawnwald E. Valvular heart disease a textbook of cardiovascular medicine, $6^{\text {th }}$ ed. New York. WB Sanders. 2001;1643.

2. Baumgartner H. et al; ESC/EACTS Guidelines for the management of valvular heart disease.
Eur Heart J. 2017;38:2739-91.

3. Gebhard MM, Preusse CJ, Schnabel PA, et al. Different effects of cardioplegic solution HTK during single or intermittent administration. Thorac Cardiovasc Surg. 1984;32:271-6.

4. Bretschneider HJ, Hübner G, Knoll D, et al. Myocardial resistance and tolerance to ischemia: physiological and biochemical basis. J Cardiovasc Surg. 1975;16:241-60.

5. Chambers DJ. Mechanisms and alternative methods of achieving cardiac arrest. Ann Thorac Surg. 2003;75:S661-6.

6. Bretschneider HJ. Myocardial protection. Thorac Cardiovasc Surg. 1980;28:295-302.

7. Fannelop T, Dahle GO, Salminen PR, et al. Multi-dose cold oxygenated blood is superior to a single dose of Bretschneider HTK cardioplegia in the pig. Ann Thorac Surg. 2009;87:1205-13.

8. Scrascia G, Guida P, Rotunno C, et al. Myocardial protection during aortic surgery: comparison between Bretschneider-HTK and cold bloodcardioplegia. Perfusion. 2011;26:427.

9. Careaga G, Salazar D, Tellez S, et al. Clinical impact of histidine-ketoglutarate-tryptophan (HTK) cardioplegia solution on the perioperative period in open heart surgery patients. Arch Med Res. 2001;32:296-9.

10. Liu J, Feng Z, Zhao J, et al. The myocardial protection of HTK cardioplegic solution on the long-term ischemic period in pediatric heart surgery. ASAIO J. 2008;54:470-3.

11. Kammerer I, Nagib R, Hipp G, et al. Myocardial Protection in Minimally Invasive Mitral Valve Surgery: Comparison of the Cold-Blood Cardioplegia of the Bretschneider Solution and the Warm-Blood Cardioplegia of the Calafiore Protocol. Arch Clin Exp Surg. 2012;1:14-21.

12. James J, Edelman B, Seco M et al. Custodiol for myocardial protection and preservation: a systematic review. Ann Cardiothorac Surg. 2013;2(6):717-28.

13. Sansone F, Punta G, Parisi F, et al. Right minithoracotomy versus full sternotomy for the aortic valve replacement: preliminary results. Heart Lung Circ. 2012;21:169-73.

14. Takeuchi K, Buenaventura P, Cao-Danh H, et al. Improved protection of hypertrophied left ventricle by histidine-containing cardioplegia. Circulation. 1995;92:395-9.

15. Paparella D, Cappabianca G, Visicchio G, et al. Cardiac troponin I release after coronary artery bypass grafting operation: effects on operative and midterm survival. Ann Thorac Surg. 2005;80:1758-64. 